\title{
On the use of wavelet analysis for the fitting of a dynamical theory to observations
}

\author{
A. Fienga ${ }^{1}$ and J. M. Delouis ${ }^{2}$ \\ 1 IMCCE, Observatoire de Paris, 77 Av. Denfert-Rochereau, 75014 Paris, France \\ 2 Observatoire de Meudon DASOP-LPSH, 92125 Meudon Cedex, France
}

Received 20 December 1999 / Accepted 6 June 2000

\begin{abstract}
We present methods to fit planetary ephemerides to observational data. More precisely, we will consider an application of wavelet analysis to dynamical residuals of Jupiter. The efficiency of this new technique is assessed by tests with extrapolations using observational samples not used in the fit. The results are very encouraging. Since the work of Gaillot in 1913 (Gaillot 1913), the analytical solutions of the Bureau des longitudes have not been fit to actual observations. The VSOPxx (Bretagnon 1982; Bretagnon \& Francou 1988) analytical theories of the motion of the 8 planets of the solar system are presently the modern solutions of the IMC-Bureau des longitudes; however, until this work, VSOPxx were fit only to the JPL numerical integrations, Dexxx (Standish 1995, 1998). This paper deals with some elements of the first adjustment of the modern VSOP analytical theories of motion of the planets to optical and radar observations.
\end{abstract}

Key words. methods: data analysis - astrometry - Ephemerides

\section{Introduction}

An adjustment of an astronomical theory should use as many observations as possible. This is true particularly for outer planets such as Jupiter, whose periods are longer than the interval since the last significant improvement in technical measurements (VLBI, Radar, etc.). In this paper, we have used older data with a higher noise level than more modern observations, in order to measure longer time variations. The first step for an accurate adjustment of orbital parameters, is to remove systematic effects, such as those of refraction effects, which exist in older catalogs. We have developed a new way to interpret differences between theories and observations in order to highlight systematic effects of particular periods associated with particular catalogs.

The search for periods of time series has been done with many methods (e.g. Cuypers 1993), but none gives enough information about possible variable spectral properties, such as changes of period, amplitude and phase. In order to interpret local and time-dependent phenomena of the data set, we need a two-dimensional timefrequency method. Wavelet Transform is such a method; it has been applied in many fields, and its general aspects have been investigated in great detail (Bijaoui 1991; Daubechies 1992; Meyer 1993, and others).

Send offprint requests to: A. Fienga, e-mail: fienga@bdl.fr
In this paper we use wavelet analysis on astronomical residual data which are very noisy and non-regularly sampled. The study of these will give information about astronomical data residuals for a given time at a given frequency. Szatmàry et al. (1994) have presented a Discrete Wavelet Transformation for the analysis of variable star light curves. They have demonstrated the importance of the data sampling and highlight the great interest in comparing Wavelet Transform on measurements and noise in order to account for sampling gap effects.

In this work, we would like to have information about absolute amplitude in order to determine what has to be corrected in the data and/or in the theory in order to decrease the residuals' amplitude. The Wavelet Transform is then normalized to measure as precisely as possible the frequency amplitude.

As a first step, we will define our wavelet transform and illustrate the analysis using simulated data. From the same computation on jovian astronomical residual data, we will extract systematic effects associated with mismeasurement. Finally, we will subtract those effects and demonstrate the efficency of such methods by fitting a preliminary analytical solution of the motion of Jupiter to these data. 


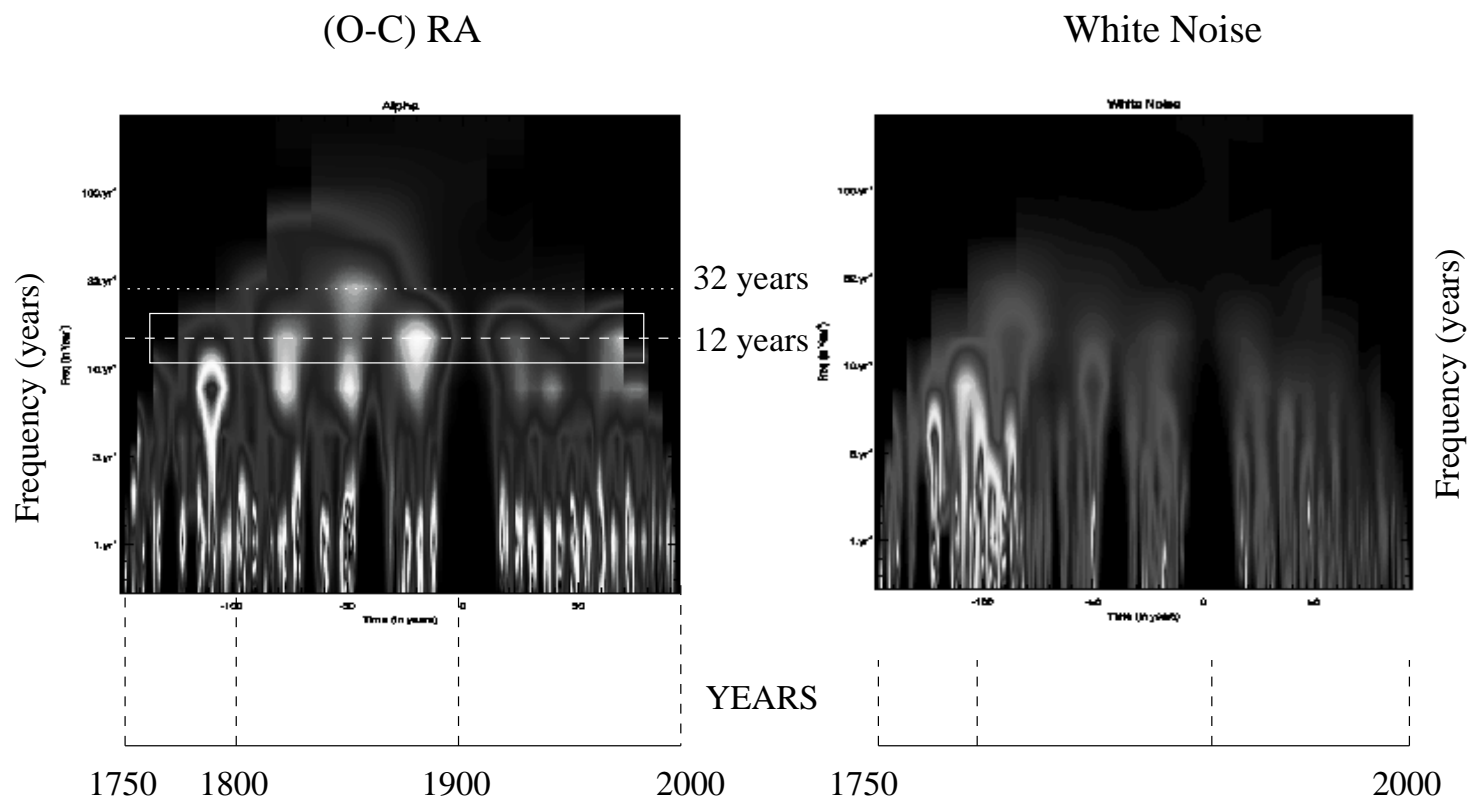

Fig. 1. Amplitude of the $W(\omega, \tau)$ function on simulated data. On the left, two periods have been added to the white noise presented on the right. One has a period of 12 years and the second, with a period of 30 years, is present only around the time 1850. On both plots, the effects of non-regular time sampling are visible (in the yellow rectangle) and appear, particularly, in white noise analysis like random variations on higher frequencies. On the left frame, one can see amplitude variation distributed regularly on a period of 12-year. A 30-year period is also detected around 1850. This shows the ability of wavelet analysis to measure amplitude fluctuation at each frequency. The effect corresponding to a 9-year period (in the yellow rectangle) and detected both in the left and right frames, is an effect induced by the non regular time distribution of the sampling. It is then considered as an artifact of the time distribution

\section{Wavelet analysis of non-regular temporal data}

\subsection{Details of the method used}

Several methods have been developed in order to compute efficiently the wavelet transform of discrete data (see, e.g. Szatmary et al. 1994). However, for our study we have simply convolved the data by a normalized wavelet filter, defined as:

$\psi_{\omega}(t)=\frac{1}{2 \sqrt{2 \pi} \theta}\left(1-\frac{t^{2}}{\theta^{2}}\right) \mathrm{e}^{1-\frac{t^{2}}{2 \theta^{2}}}$,

where $\theta=\frac{1}{\sqrt{2} \pi \omega}$. The first coefficient is normalized in order to have a wavelet coefficient equal to the amplitude of a sinusoidal function with a period equal to $\omega$. In such a scheme, the wavelet coefficient for a time series will correspond to the amplitude of the detected frequency. Following Slezak et al. (1990), the whole frequency domain can be scanned from the cutoff frequency $\nu_{0}$ (the average time sampled value) to the frequency corresponding to the half data period. Practically, $\omega=\nu_{0} 2^{n}$, where $n$ is an integer greater or equal to 0 and whose maximal value is that which gives $\omega_{\max }$ equal to half the data period. Finally, we adopt, for a non-regular time distribution, a $W(\omega, \tau)$ wavelet coefficient defined by:

$W(\omega, \tau)=\Delta t \sum_{j}\left(h_{j}-\bar{h}\right) \psi_{\omega}\left(t_{j}-\tau\right)$,

where $j$ ranges over the number of observations, $t_{j}$ is the time of the observation $j, h_{j}$ is the observational value studied in this computation, $\bar{h}$ is the average value of $h_{j}$ over the complete data set, and $\Delta t=\frac{1}{N} \sum_{j}\left(t_{j+1}-t_{j}\right)$ is the average time sample of the time series.

This expression of $W(\omega, \tau)$ does not give an amplitude of fluctuation. As described by Abergel et al. (1996), we define the amplitude of fluctuation at a particular time $\tau$ as the rms fluctuation from $\tau-\theta$ to $\tau+\theta$. All figures in this paper are computed from that function of $W(\omega, \tau)$.

It is then clear that both the time series variation and noise fluctuations contribute to this final result. As such, a user should compensate for this by comparing results from the analyzed time series with those obtained from white noise having a similar amplitude.

\subsection{Results on simulated data}

In order to show the efficiency of our wavelet analysis, we have checked it using simulated data. One of the features we wish to highlight in this presentation is the effect of non-regular sampling. We simulate astrometrical observations of Jupiter containing systemtic signatures with two periods, and we obtain non-regular time samples for analysis. Figure 1 shows the efficiency of our wavelet analysis which detects the two frequencies in our simulated data.

The two periods have their amplitude 3 times higher than the noise rms. By comparing the results from Wavelet Transform on this simulation with the results from a white background noise, we succeed in detecting all frequencies. This would have not been possible with a classic Fourier 
transform. More precisely, the background noise wavelet transform helps us recognize the artificial effects due to non-regularity of the sampling. For example, in Fig. 1, a 9 -year effect can be seen (yellow rectangle on the maps). However, this effect also appears in the white noise wavelet transform, implying that this effect is not a real effect induced by the observations but an artifact due to the non-regular time distribution of the sampling. Thanks to the technique of comparison, it is possible to discriminate between the real effects, which clearly appear only on the $(\mathrm{O}-\mathrm{C})$ wavelet transforms, and the artificial effects, which are also in the background noise wavelet transform. Therefore, we have the opportunity to measure for a particular frequency, such as the 30-year period, its amplitude variation. The fact that some fluctuations are present for the 12-year period is due to non-regular time sampling.

\subsection{Comparison with Fourier analysis}

In order to demonstrate the difference between wavelet and Fourier analysis, we have computed the periodogram of the same data set. This result is shown in Fig. 2. A periodogram is certainly less affected by the level of noise, but it does not give any information about the amplitude variation of a given frequency.

In that particular case, the periodogram shows that the 30-year period has an amplitude 10 times lower than the 10 -year period. On the contrary, the wavelet analysis found similar amplitudes for the 10- and 30-year period around 1850. Because the periodogram gives an average evaluation, it produces amplitude mis-measurements. This type of information is available only from wavelet analysis. Furthermore, wavelet analysis also provides information about the intervals of time in which the detected systematic effects are produced. This type of information is not available from a classic Fourier analysis.

If a frequency is present only during a narrow period, it appears on the periodogram as a family of frequencies. From results obtained with a wavelet analysis we have shown the ability of this process to detect such an amplitude variation. From that example we demonstrate that wavelet analysis can detect various signal parameters that Fourier analysis cannot detect.

\section{Wavelet analysis on Jupiter ephemerides}

In this paper, we make a new estimation of systematic errors in a wide-spread sample of observations of Jupiter (1750-1997), and study the impact of such an improvement on an adjustment of the general analytical solution for eight planets of the solar system, VSOP87 (Bretagnon \& Francou 1988). Thanks to wavelets analysis, it is possible to identify the time distribution and the shape of the effects that would not be detected with a classic Fourier analysis.

To demonstrate the efficency of such an approach, we apply the following analysis: first, we fit the analytical theory to our complete observational database and produce

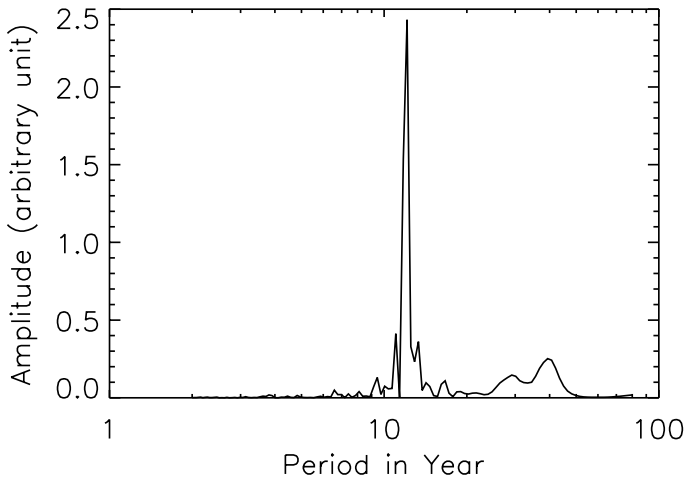

Fig. 2. Fourier analysis of simulated data. The plot shows perfectly the presence of a 12-year period, but does not detect correctly the 30-year period. This type of process could not detect amplitude variation for a single frequency. Furthermore, we do not have any information about the periods of time which induce these effects

what we call "base residuals" from the resulting adjusted ephemeris. This database covers the interval, 1750-1997, and contains a wide variety of observations; very accurate angular positions of Jupiter deduced from VLBI tracking data of the Galileo spacecraft (Folkner 1996-98), accurate positions of the planet deduced from photographic observations of its satellites and reduced with Hipparcos stars (Fienga 1998), and various transit observations taken at different observatories between 1750 and 1982 (Paris, Greenwich, and Washington). For more details, see Fienga (1999). Second, we obtain a second solution, one from which we have excluded certain sets of observations; from these, we compute new residuals for the data that we have excluded from the fit, calling these results without corrections. Third, we perform a number of wavelet analyses on the reduced observational sample, using different possible correction models. Fourth, we perform an adjustment of the analytical theory to this wavelet-corrected sample and obtain new residuals for the excluded observations (results with corrections).

Therefrom, it is possible to estimate the impact of the wavelet correction. Our results show the improvement of the wavelet-corrected residuals over the uncorrected residuals. The excluded observations provide a good test; they are accurate positions of Jupiter deduced from satellite observations taken during the Hipparcos and Tycho missions (ESA 1997) and the accurate 1985-96 transit observations of the La Palma Observatory with the Carlsberg Meridian Circle (Morrison 1997).

\subsection{Detection of systematic effects}

We have applied the wavelet analysis on post-fit Jupiter residuals for a period of two centuries. As shown in Fig. 3, a 12-year period (sideral period of Jupiter) structure is detected in right ascension, as in declination. There are also significant structures at 32- and 100-year periods. 

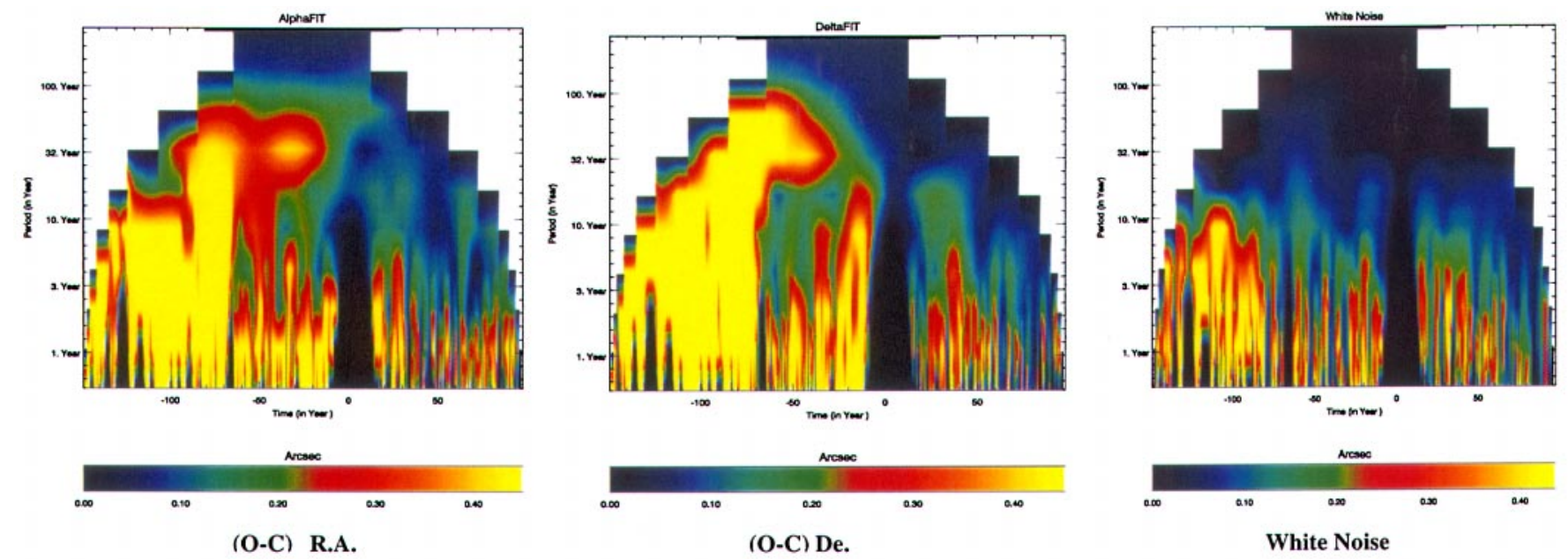

Fig. 3. Wavelet analysis of post-fit residual data in right ascension (left frame) and declination (central frame), compared to the same analysis on a simulated white noise (right frame). Significant structure are clearly visible on long periods
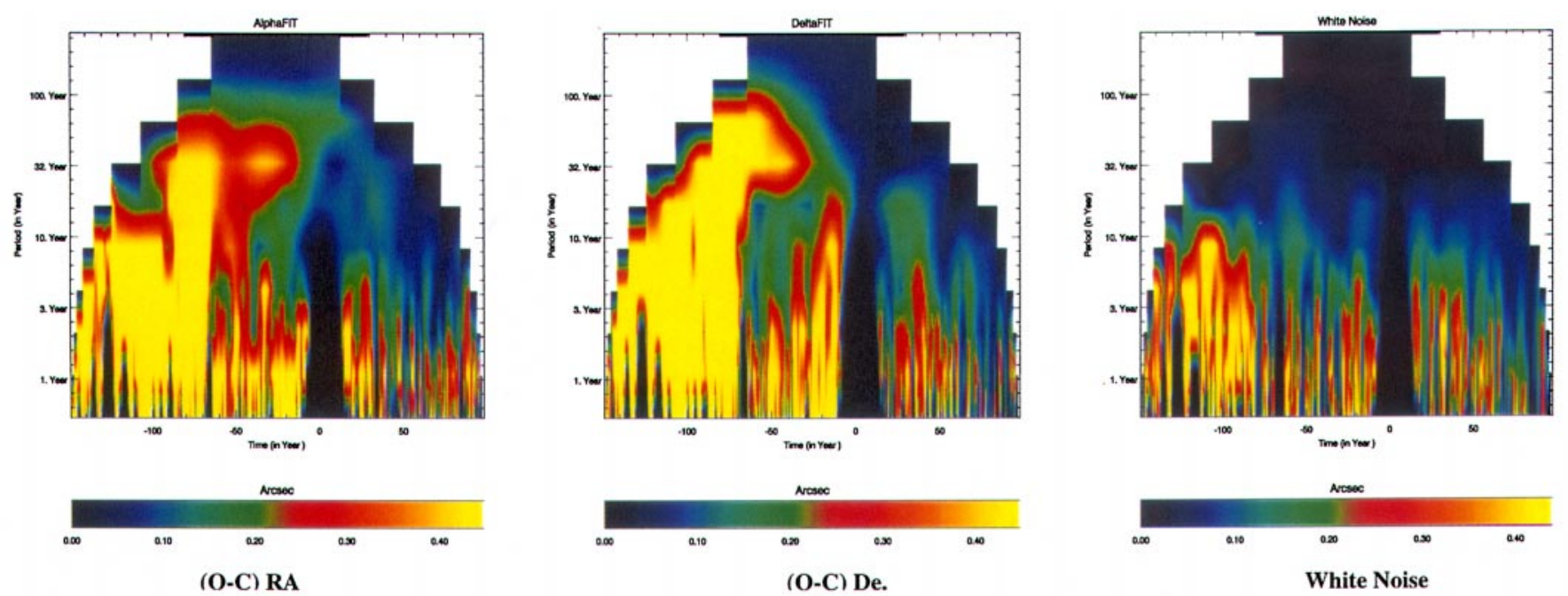

Fig. 4. Wavelet analysis of post-fit residual data in right ascension (left frame) and declination (central frame), compared to the same analysis on a simulated white noise (right frame). This results come from a comparison between observed positions between 1750 and 1994 and computed from DE405

By comparing this result with white noise data for a similar time distribution, we can determine which structures are linked to non-continuous time acquisition and which structures result from actual systematic observational effects. For example, see Fig. 1. From this first step analysis, we have demonstrated the existence of systematic structures. Other frequencies ( $<9$-year) are not significant compared to the ones from aliasing due to non-regular data sampling.

\subsection{Comparison between theories}

We have checked to see if the detected frequencies have been induced by a bad adjustment of the analytical solution or by the dynamics itself. This we have done by performing wavelet analysis on the same data using both VSOP87 and DE405 (Standish 1998), as shown in Figs. 3 and 4 , respectively. The similarity between the two sets of plots is evident. We also note that VSOP87 fit onto DE200 and DE405 were directly or indirectly fit onto the same sample of observations. Therefore, we have detected effects which are linked to observational methods or reduction processes and not due to the ephemeris adjustment. We have next tried to determine which observational errors can produce those phenomena.

\subsection{Correction determination}

It is clear that regional or zonal errors linked to a stellar catalog from ground-based observations can introduce periodical effects equivalent to the sideral period of the measured object. For instance, every 12 years, Jupiter is observed in a similar sky direction, so that its position is shifted by positional errors of reference stars used for that sky region. For observations done at higher or lower declination, the residuals are higher than at other 
declinations. This effect is even bigger for older data. This analysis supports the hypothesis that systematic effects result from zonal errors. Furthemore, it is possible to see three distinct intervals in the observational data: before 1820, 1820-1900 and after 1900. This detection of a systematic effect over a specific period is a direct result of the wavelet analysis, not easily obtainable from a classical Fourier analysis.

On the other hand, atmospheric refraction models have been modified during the past two centuries: adopted corrections for observations have evolved as a result of better atmospheric modeling and improved instrumentation.

\subsection{Subtraction of systematic effects}

Before the adjustment we have checked different hypotheses to explain systematic errors of Jupiter's positions. We have divided the data into three periods. The first contains data before 1820; the second, data between 1820 and 1910; and the last, data from 1910 to 1940 . The last period is limited to 1940 because it appears that modern data sets do not present significant errors. On those three data periods, we have adjusted different types of corrections, corresponding to evolving refraction knowledge and to systematic error from reference star catalogs. These corrections were done one by one and are not independent. For each correction, we have tested different models. We will present here only those which give the best results.

\subsubsection{Refraction effect}

This correction is zenithal, and for transit observations, affects only declination measurements. We have used the classical Laplace model:

$$
(\mathrm{O}-\mathrm{C})_{\mathrm{DZ}}=A \tan D Z+B \tan ^{3} D Z
$$

and have adjusted the parameters, $A$ and $B$. Our aim is to remove systematic effects from the observations. These corrections were done only for the last period (1910-1940), because the older data noise level is too high to compute adjusted parameters.

In comparing maps $\mathrm{B}$ and $\mathrm{C}$ shown in Fig. 5, where one is corrected $(\mathrm{C})$ and the other is not $(\mathrm{B})$, we notice the subtraction of systematic effects in declination. This indicates that the data had not been well-corrected for refraction, because of modeling errors or mis-interpretation of meteorological conditions. Some trends seem to appear or disappear in right ascension even though the right ascension coordinates are independent of the refraction corrections in transit observations. These trend modifications are induced by combinations (or correlations) between zonal and refraction corrections, the fit of these models being computed simultaneously on the same sample.

\subsubsection{Zonal effect due to star catalogs}

After a certain number of tests, we have adopted two types of model. One will be called complex and is similar to a Fourier series, patterned after Schwan (1977):

$$
\left\{\begin{array}{l}
(\mathrm{O}-\mathrm{C})_{\alpha}=\sum_{i=0}^{k} C_{i+1} \cos i \delta \\
(\mathrm{O}-\mathrm{C})_{\delta}=D_{1}+\sum_{i=1}^{k} D_{i+1} \sin i \delta
\end{array}\right\},
$$

where $C_{i}$ and $D_{i}, i=1 \ldots k$, are parameters which are adjusted from data. From this adjustment, we have a more accurate correction of systematic effects in right ascension and declination. The plot $\mathrm{C}$ in Fig. 5 shows that the correction by complex models is sufficient to remove systematic effects for the 32- and 100-year periods. It confirms that observations from 1820 to 1900 are not completely corrected by this process and that other corrections are necessary. However, for observations done between 1910 and 1940, the corrections increase the signal in declination and in right ascension. This is due to the fact that this correction had already been applied. So, by computing this correction a second time, we have interpreted noise as signal and, as a result, we have amplified the systematical errors. For this reason, we have tried another correction for that period; we have called it the simple model, chosen empirically, motivated by the appearance of the wavelet maps:

$$
\left\{\begin{array}{c}
(\mathrm{O}-\mathrm{C})_{\alpha}=\sum_{i=0}^{k} E_{i+1} \delta^{i} \\
(\mathrm{O}-\mathrm{C})_{\delta}=\sum_{i=0}^{k} F_{i+1} \delta^{i}
\end{array}\right\}
$$

where $E_{i}$ and $F_{i}, i=1 \ldots k$ are parameters which are adjusted from the data. Results of that analysis are presented in plots D of Fig. 5. They show that the effects linked to the model induced by the Fourier series have been removed. This polynomial method is then used to correct periods in order to complete other corrections.

\section{Test by fitting}

We have computed two sets of residuals for the data not included in the adjustment - one from the original base solution and the other from the adjusted solution - and we have compared the two sets. The non-included data types are different in time and technology - ground-based meridian circle observations on the one hand and spatial scanning missions on the other hand -, which is why the noise level is very different between these two periods, 1986-1995 and 1990-1994. These results are presented in Table 1.

We have presented results from older solutions (DE405, VSOP87). Morrison \& Evans (1998) have also studied such results, finding that optical positions compared to positions deduced from DE405 all show a positive bias in right ascension, regardless of the jovian satellite that was observed. In particular, the Hipparcos points for Europa have an average value of +20 mas. In declination, Hipparcos observations have an average offset of -20 mas. We also note an important systematic trend 


\section{Solution before treatment}

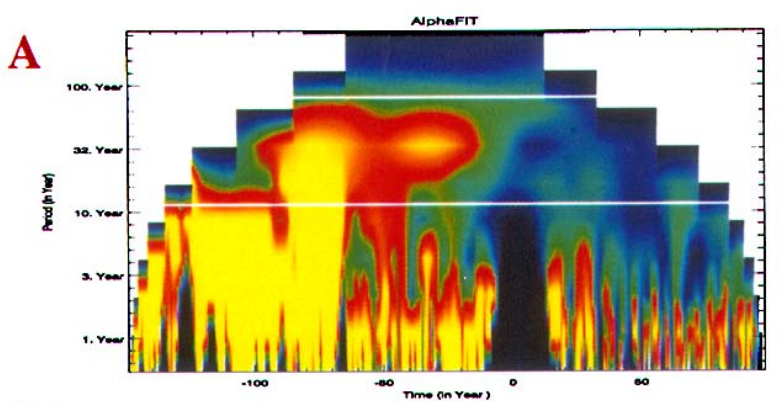

RA
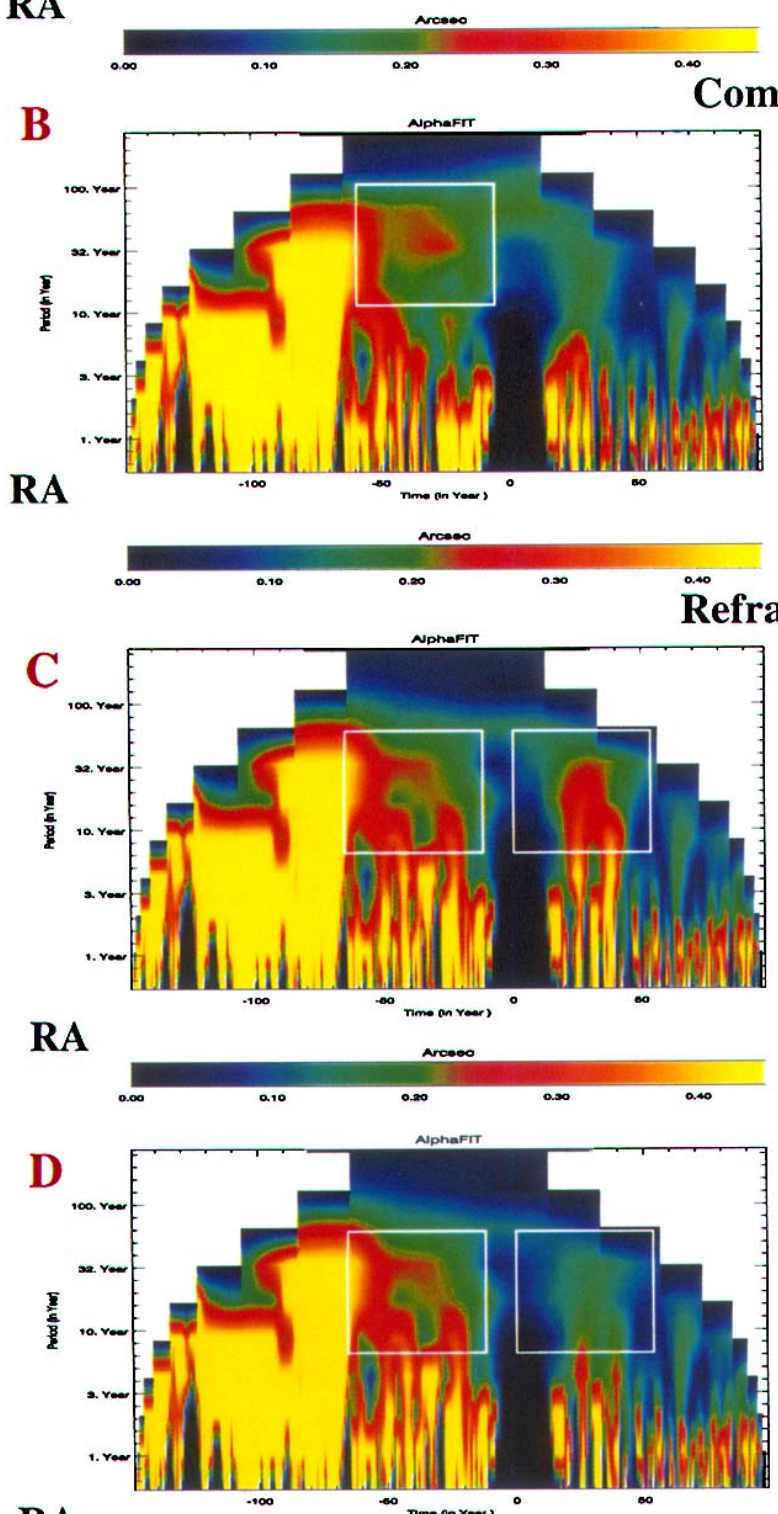

RA

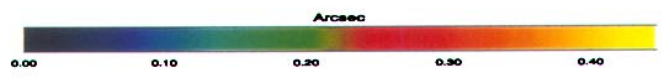

De
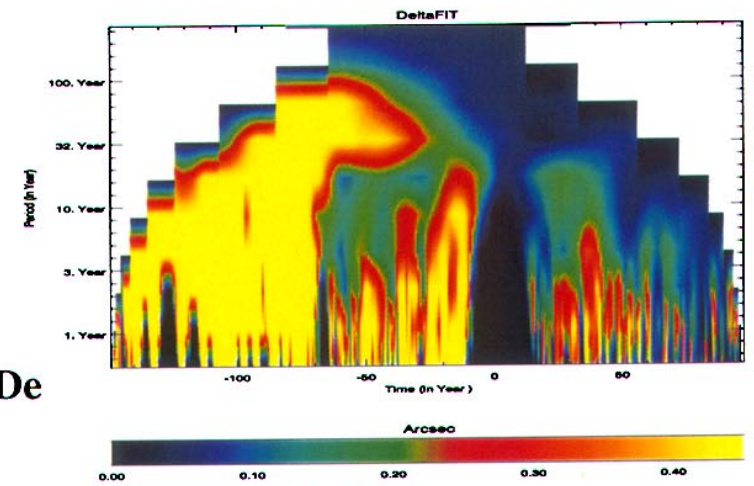

$\stackrel{0.00}{0.10}$ Corrections only $^{0.00}$

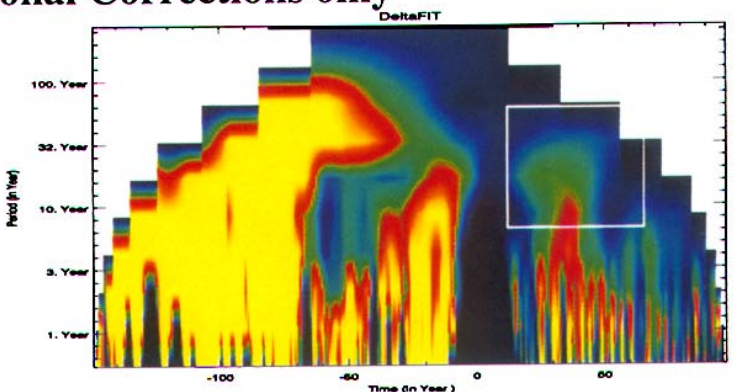

De

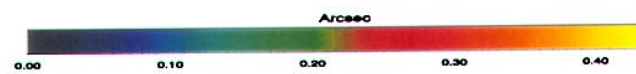

ection

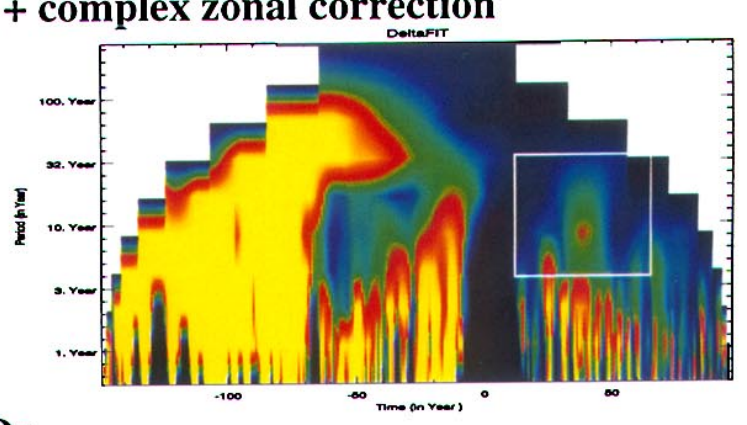

De
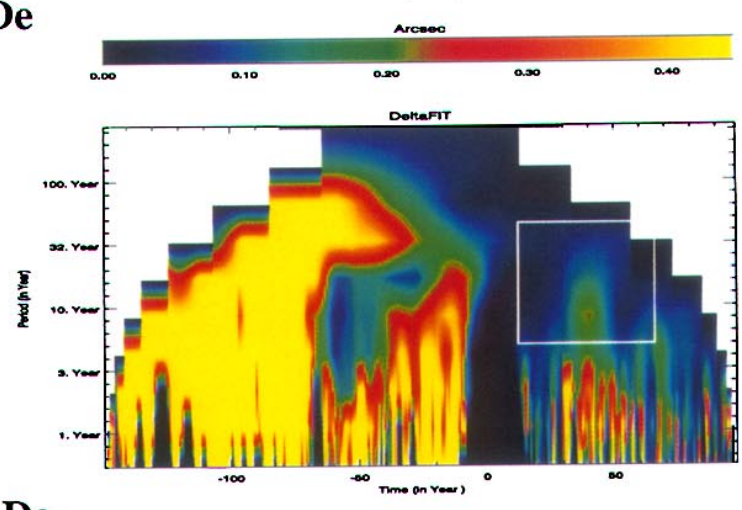

De

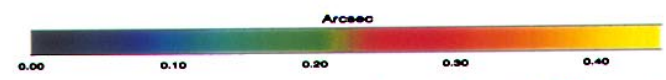

Refraction + complex zonal correction for 1900 period + simple zonal correction for $1800-1700$ period

Fig. 5. Wavelet analysis of residual data for right ascension (right column) and declination (left column), after adjustment of the initial condition of jovian movement. Observations were corrected for different effects: complex zonal corrections, refraction correction, and simple zonal correction (see text for details). The first analysis is shown without treatment, corresponding to an analysis done after adjustment and without any correction of observational effects 
Table 1. Prediction result from different adjusted solution from various corrected observations. Presented in this table are the residuals (in arcseconds) computed by comparison between observed positions of the center of mass of Jupiter (from La Palma and from the Hipparcos mission) and computed positions deduced from VSOP87, DE405 and our newly fit solutions. The observations are corrected for refraction and zonal effects by a complex (solution C) or simple (solution D) formulation. These two adjusted solutions are based upon a radar and VLBI Earth-Moon barycenter motion

\begin{tabular}{|c|c|c|c|c|c|c|}
\hline \multirow[t]{2}{*}{ JUPITER } & \multicolumn{2}{|c|}{ CAMC (1986-1995) } & \multicolumn{2}{|c|}{ Tycho (1990-1994) } & \multicolumn{2}{|c|}{ Hipparcos (1990-1994) } \\
\hline & (OC) RA & (OC) De. & $(\mathrm{OC}) \mathrm{RA}$ & (OC) De. & $(\mathrm{OC}) \mathrm{RA}$ & (OC) De. \\
\hline VSOP87 & $+0.081 \pm 0.207$ & $-0.012 \pm 0.241$ & $-0.037 \pm 0.088$ & $-0.054 \pm 0.050$ & $-0.010 \pm 0.009$ & $+0.014 \pm 0.005$ \\
\hline DE405 & $+0.062 \pm 0.207$ & $-0.013 \pm 0.220$ & $-0.020 \pm 0.089$ & $-0.024 \pm 0.044$ & $+0.017 \pm 0.005$ & $-0.021 \pm 0.003$ \\
\hline \multicolumn{7}{|l|}{ 1914-1997 } \\
\hline & $+0.043 \pm 0.206$ & $-0.002 \pm 0.222$ & $-0.015 \pm 0.089$ & $-0.031 \pm 0.045$ & $+0.023 \pm 0.009$ & $-0.026 \pm 0.008$ \\
\hline \multicolumn{7}{|l|}{$1750-1997$} \\
\hline & $+0.071 \pm 0.200$ & $-0.031 \pm 0.245$ & $-0.011 \pm 0.089$ & $-0.032 \pm 0.045$ & $+0.025 \pm 0.006$ & $-0.027 \pm 0.013$ \\
\hline \multicolumn{7}{|l|}{ Solution C } \\
\hline \multirow[t]{2}{*}{ Solution D } & & & & & & \\
\hline & $+0.026 \pm 0.202$ & $-0.008 \pm 0.217$ & $-0.012 \pm 0.089$ & $-0.036 \pm 0.045$ & $+0.025 \pm 0.007$ & $-0.029 \pm 0.004$ \\
\hline
\end{tabular}

of VSOP87 solution compared to DE405. This trend is not only detectable in right ascension, but also in the dispersion of the declination residuals. The intrinsic quality of that analytical solution and the solution of planetary masses used in VSOP87 (UAI, 1976) can possibly explain this discrepancy. Bretagnon \& Francou (1988) estimate that the accuracy of the VSOP 87 theory of the motion is about 20 mas for the observational period considered here (1985-1995).

Secondly, we have presented the statistics of the residuals obtained after comparison between observed positions (CAMC, Tycho and Hipparcos observations) and positions deduced from our new fitted solutions. The two first (called 1914-1997 and 1750-1997) are based on the shorter (1914-1997) and the longer (1750-1997) observational series used for fitting. These series are not waveletcorrected. These two solutions (1914-1997) and (17501997) improve the prediction capability of the VSOP87 solution. The solutions called Solution $C$ and Solution D correspond to solutions fitted on a sample of observations performed between 1750 and 1997 but corrected for effects detected with the wavelets analysis. As described before, two correction models for zonal errors and errors due to poor refraction correction were applied, resulting in 2 solutions, Solution $C$ and Solution D. The residuals obtained by comparisons between observed positions and positions deduced from the fitted solutions $\mathrm{C}$ et D are also presented in Table 1 . They are very close to those obtained prevously. All the results presented in Table 1 imply firstly that it's possible to improve the capability of prediction of the VSOP87 solution of the motion of Jupiter by fitting its constants of integration to old and modern observations (solutions (1914-1997) and (1750-1997)), and secondly that no peculiar trend is induced by our wavelet treatment degrading the prediction capabilty of the fitted solution.

\section{Conclusion}

Because of abilities of wavelet transform to detect structures and amplitudes of systematic errors inside data sets, we have tried to use it for astronomical applications. From the development of an algorithm which gives an accurate estimation of the amplitude variation at one frequency, we have succeeded in detecting and even in removing some refraction and zonal effect from older data. It gives us then the opportunity to adjust astronomical solutions over a large data set, particularly for longer periods than previously were possible.

In the future, the use of this method to adjust data for other planets, or on other types of data, will provide the opportunity to correct systematical effects and to merge old and new data, which were often not reduced by similar processes. Therefore, using accurate planetary masses (IERS, 1992) and more developed perturbations, the fit to observations with a new analytical solution is very promising.

Acknowledgements. We would like to send special thanks to Dr. Standish for numerous and very helpful discussions.

\section{References}

Abergel, A., Boulanger, F., Delouis, J. M., Dudziak, G., \& Steindling, S. 1996, A\&A, 309, 245

Bijaoui, A. 1991, Data Analysis Workshop, 3

Bretagnon, P. 1982, A\&A, 114, 278

Bretagnon, P., \& Francou, G. 1988, A\&A, 202, 309 
Cuypers, J. 1993, in Applications of Time Series Analysis in Astronomy and Meteorology, ed. O. Lessi, Padova, Italy, 139

Daubechies, I. 1992, Ten Lectures on Wavelets, June 1990, Philadelphia: SIAM (Society for Industrial and Applied Mathematics)

ESA 1997, in ESA, SP-1200

Fienga A. 1998, A\&A, 335, 1111

Fienga, A. 1999, Ph.D. Thesis, Observatoire de Paris

Folkner, W. M. 1996-98, private communications

Gaillot, A. 1913, Ann. Obs. Paris, 31

IERS Standards 1992, ed. D. D. McCarthy

Meyer, Y. 1993, Philadelphia: SIAM (Society for Industrial and Applied Mathematics)
Morrison, L. V. 1997, Carlsberg Meridien Circle, La Palma, vol. 9

Morrison, L. V., \& Evans, D. W. 1998, A\&A, 132, 381

Standish, E. M. 1995, Technical report, Jet Propulsion Laboratory Interoffice Memorandum

Standish, E. M. 1998, Technical report, Jet Propulsion Laboratory Interoffice Memorandum

Slezak, E., Bijaoui, A., \& Mars, G. 1990, A\&A, 227, 301

Schwan, H. 1977, Veroeffentlichungen des Astronomishen Rechen-Institut, Heidelberg, 27

Szatmary, K., Vinko, J., \& Gal, J. 1994, A\&A, 108, 377

UAI Transactions, 1976, vol. XVIB 52 